\title{
STRICTLY POSITIVE AND STRONGLY POSITIVE SEMIGROUPS
}

\author{
ADAM MAJEWSKI and DEREK W. ROBINSON
}

(Received 12 August 1981)

Communicated by J. B. Miller

\begin{abstract}
We examine positive semigroups acting on Banach lattices and operator algebras. In the lattice framework we characterize strict positivity and strict ordering of holomorphic semigroups by irreducibility criteria. In the algebraic setting we derive ergodic criteria for irreducibility and discuss various aspects of strict positivity. Finally we examine invariant states of a $C^{*}$-dynamical system in which the automorphism group is replaced by a strongly positive semigroup. We demonstrate that ergodic states are characterized by a cluster property despite the absence of a covariant implementation law for the semigroup.
\end{abstract}

1980 Mathematics subject classification (Amer. Math. Soc.): primary 47 D 05; secondary 47 C 15.

\section{Introduction}

In several recent papers, Bratteli, Kishimoto and Robinson (1980), Kishimoto and Robinson (1981), Bratteli and Robinson (1981a), various aspects of positive semigroups acting on function spaces and operator algebras were examined. The first purpose of the present note is to extend to Banach lattices some of these results which characterize strictly positive holomorphic semigroups on $L^{p}$-spaces. Secondly we discuss various aspects of positive and strictly positive semigroups acting on operator algebras. Finally we examine the ergodic structure of invariant states over $C^{*-}$ (and $W^{*}$ ) dynamical systems formed by an algebra and a strongly positive semigroup acting on the algebra.

Written while the first author was on leave of absence from Gdansk University.

c Copyright Australian Mathematical Society 1983 


\section{Banach lattices}

A (real) Banach lattice is a Banach space 9 equipped with an order relation $\geqslant$ such that $\mathscr{B}$ is a lattice with respect to the order and, moreover, the ordering respects the topological structure. In particular if $x, y \in \mathscr{B}$ and $|x| \leqslant|y|$ then $\|x\| \leqslant\|y\|$ where $|\cdot|$ denotes the modules of an element and $\|\cdot\|$ denotes the norm. For further details about Banach lattices we refer to Schaefer (1974). Although we deal with real lattices all our results can be extended to complex lattices by the usual complexification procedure.

Next let $S=\left\{S_{t}\right\}_{t \geqslant 0}$ be a $C_{0}$-semigroup acting on the Banach lattice $(9 \mathfrak{B}, \geqslant)$. We define $S$ to be positive, and write $S \geqslant 0$ if $S_{t} \Re_{+} \subseteq \mathscr{G}_{+}$for all $t>0$ where $\mathscr{B}_{+}$ denotes the positive elements of $\mathscr{B}$. Moreover we define $S$ to be strictly positive, $S>0$, whenever

$$
f\left(S_{t} x\right)>0
$$

for all non-zero $x \in \mathscr{B}_{+}$, all non-zero $f \in \mathscr{B}_{+}^{*}$, and all $t>0$.

We begin by recalling a result which connects strict positivity and an irreducibility condition. Basically a set of bounded operators acting on some structure is said to be irreducible if it does not leave any proper substructure invariant. Emphasis on different structural features leads to different notions of irreducibility and it is important to distinguish between them.

If $A$ is a set of bounded operators on a Banach space $\mathscr{B}$ then a subset $S \subseteq \mathscr{B}$ is called $A$-invariant if $A S \subseteq S$. Moreover $A$ is defined to be $B$-irreducible if $\{0\}$ and $\mathscr{B}$ are the only $A$-invariant Banach subspaces.

Next a subspace $S \subseteq \mathscr{B}$ is called solid if $x \in S, y \in \mathscr{B}$, and $|y| \leqslant|x|$, implies $y \in S$. Equivalently $S$ is solid if $1 . x \in S$ implies $|x| \in S$, and 2. $x \in S_{+}$, $y \in \mathscr{B}_{+}$, and $0 \leqslant y \leqslant x$ implies $y \in S_{+}$. A solid subspace of $\mathscr{Q}$ is called an (order) ideal and a solid Banach subspace is called a Banach ideal. Finally the set of bounded operators $A$ is defined to be $B I$-irreducible if $\{0\}$ and 9 are the only $A$-invariant Banach ideals.

The following criteria for $B I$-irreducibility of a positive semigroup follows from Chapter III, Proposition 8.3, in Schaefer (1974).

Proposition 1. Let $S=\left\{S_{t}\right\}_{t \geqslant 0}$ be a positive semigroup acting on the Banach lattice $(G, \geqslant)$. The following conditions are equivalent:

1. $S$ is BI-irreducible,

2. for each $x>0$ the ideal generated by $\left\{S_{t} x\right\}_{t>0}$ is dense in $\mathscr{B}$,

3. for each non-zero $x \in \mathscr{B}_{+}$and non-zero $f \in \mathscr{B}_{+}^{*}$ there exists a $t>0$ such that $f\left(S_{t} x\right)>0$. 
Next we equate $B I$-irreducibility of a holomorphic semigroup with strict positivity of the semigroup by extension to Banach lattices of a result previously obtained for $L^{p}$-spaces, Kishimoto and Robinson (1981),

Proposition 2. Let $(\mathscr{B}, \geqslant)$ be a Banach lattice and $S=\left\{S_{t}\right\}_{t \geqslant 0}$ a positive holomorphic semigroup acting on $\mathscr{B}$. If $x \in \mathscr{B}_{+}$and $f \in \mathscr{B}_{+}^{*}$ then either

$$
f\left(S_{t} x\right)>0 \text { for all } t>0 \text {, }
$$

or

$$
f\left(S_{t} x\right)=0 \quad \text { for all } t>0
$$

Proof. The proof is very similar to that of Simon (1973), Kishimoto and Robinson (1981), but one needs to emphasize various features of the lattice structure. First choose a sequence $t$ such that $t_{n} \rightarrow 0$ and

$$
\left\|x-S_{t_{n}} x\right\| \leqslant 2^{-n} \text {. }
$$

Next for $m \leqslant n$ define $x_{m, n}$ by

$$
x_{m, n}=x \wedge\left(\wedge_{m \leqslant k \leqslant n} S_{t_{k}} x\right) .
$$

It follows that

$$
\begin{aligned}
0 & \leqslant x_{m, n}-x_{m, n+1} \\
& =x_{m, n} \wedge\left(x-S_{t_{n+1}} x+S_{t_{n+1}} x\right)-x_{m, n} \wedge S_{t_{n+1}} x \\
& \leqslant x_{m, n} \wedge\left(\left(x-S_{t_{n+1}} x\right)_{+}+S_{t_{n+1}} x\right)-x_{m, n} \wedge S_{t_{n+1}} x \\
& \leqslant x_{m, n} \wedge\left(x-S_{t_{n+1}} x\right)_{+} \leqslant\left(x-S_{t_{n+1}} x\right)_{+}
\end{aligned}
$$

where $y_{ \pm}$denotes the positive and negative parts of $y \in 9$ and the fourth step uses the lattice version of the triangle inequality (Schaefer (1974), page 53). Consequently

$$
\left\|x_{m, n}-x_{m, n+1}\right\| \leqslant\left\|x-S_{t_{n+1}} x\right\| \leqslant 2^{-(n+1)} .
$$

Similarly one finds that $\left\|x-x_{m, m}\right\| \leqslant 2^{-m}$ and hence

$$
\left\|x-x_{m, n}\right\| \leqslant\left\|x-x_{m, m}\right\|+\sum_{k=m}^{n-1}\left\|x_{m, k}-x_{m, k+1}\right\| \leqslant 2^{-m+1} .
$$

This method of estimation also gives $\left\|x_{m, n_{1}}-x_{m . n_{2}}\right\| \leqslant 2^{-n_{1}}$ for all $n_{2}>n_{1}$ and this last estimate shows that the limit

$$
x_{m}=\lim _{n \rightarrow \infty} x_{m, n}
$$


exists. Moreover one has $0 \leqslant x_{m} \leqslant x$ and

$$
\left\|x-x_{m}\right\| \leqslant 2^{-m+1} \text {. }
$$

Now suppose that $f\left(S_{t_{0}} x\right)=0$ for some $t_{0}>0$. Since $0 \leqslant x_{m} \leqslant x_{m, n} \leqslant S_{t_{n}} x$ for $n \geqslant m$ and $S_{t} \mathscr{B}_{+} \subseteq \mathscr{B}_{+}$one has

$$
0 \leqslant f\left(S_{t} x_{m}\right) \leqslant f\left(S_{t+t_{n}} x\right)
$$

for $n \geqslant m$. Consequently $f\left(S_{t} x_{m}\right)=0$ for $t=t_{0}-t_{n}$ with $n \geqslant m$ sufficiently large that $t_{0}>t_{n}$. Thus $t_{0}$ is an accumulation point of zeros of the holomorphic function $t \mapsto f\left(S_{t} x_{m}\right)$. Hence $f\left(S_{t} x_{m}\right)=0$ for all $t>0$ and, by limiting, $f\left(S_{t} x\right)=0$ for all $t>0$.

Combination of Propositions 1 and 2 immediately yields the following criterion for strict positivity.

THEOREM 3. Let $S=\left\{S_{t}\right\}_{t \geqslant 0}$ be a positive holomorphic semigroup acting on the Banach lattice $(\mathscr{B}, \geqslant)$. The following conditions are equivalent:

1. $S$ is strictly positive,

2. $S$ is BI-irreducible.

REMARKs. 1. The holomorphy of $S$ is only required to prove $2 \Rightarrow 1$.

2. Explicit examples show that strict positivity does not imply $B$-irreducibility.

The foregoing irreducibility criterion for strict positivity differs from that obtained in Kishimoto and Robinson (1981). For example Theorem 3 of this reference established that a positive holomorphic semigroup on $\mathscr{B}=L^{p}(x ; d \mu)$, with $1 \leqslant p \leqslant \infty$, is strictly positive if, and only if, $\left\{S_{t}\right\}_{t \geqslant 0} \cup L^{\infty}(x ; d \mu)$ is $B$-irreducible or, equivalently, if $S_{t} \cup L^{\infty}(x ; d \mu)$ is $B$-irreducible for some $t>0$. In these criteria $L^{\infty}(x ; d \mu)$ is viewed as a set of bounded multiplication operators on $L^{p}(x ; d \mu)$ and $B$-irreducibility of the larger set $S \cup L^{\infty}$ replaced $B I$-irreducibility of $S$. It is natural to ask whether there is an analogue of these results for a general Banach lattice and one problem is to find a replacement for the abelian algebra $L^{\infty}$ of multiplication operators. If $g$ is order complete the natural candidate appears to be the abelian algebra generated by the band projections, that is, the projections onto the order complete Banach ideals of $\mathscr{B}$ (see Schaefer (1974) Chapter II, Section 2 for a discussion of the existence of these projections).

Proposition 2 and Theorem 3 have analogues for the comparison of semigroups. If $S=\left\{S_{t}\right\}_{t \geqslant 0}$, and $T=\left\{T_{t}\right\}_{t \geqslant 0}$ are two semigroups on $(\mathscr{B}, \geqslant)$ then $S$ is defined to be larger than $T, S \geqslant T$, if $\left(S_{t}-T_{t}\right) \mathscr{B}_{+} \subseteq \mathscr{B}_{+}$for all $t>0$. Moreover $S$ is defined to be strictly larger than $T, S \succ T$, whenever

$$
f\left(\left(S_{t}-T_{t}\right) x\right)>0
$$


for all non-zero $x \in \mathscr{B}_{+}$, all non-zero $f \in \mathscr{B}_{+}^{*}$, and all $t>0$. The analogue of Proposition 2 is the following.

Proposition 4. Let $(\mathscr{G}, \geqslant)$ be a Banach lattice and $S=\left\{S_{t}\right\}_{t>0}, T=\left\{T_{t}\right\}_{t>0}$, two positive holomorphic semigroups acting on $\mathscr{B}$ such that $S \geqslant T$. If $x \in \mathscr{B}_{+}$and $f \in \Re_{+}^{*}$ then either

$$
f\left(\left(S_{t}-T_{t}\right) x\right)>0 \text { for all } t>0
$$

or

$$
f\left(\left(S_{t}-T_{t}\right) x\right)=0 \text { for all } t>0 .
$$

Proof. The proof is a repetition of reasoning used to prove Theorem 10 in Kishimoto and Robinson (1981).

The next result is a generalization of Theorem 3. Again the proof is a repetition of reasoning already used to obtain a similar conclusion in the $L^{p}$-context in the foregoing reference.

THEOREM 5. Let $S=\left\{S_{t}\right\}_{t>0}, T=\left\{T_{t}\right\}_{t>0}$, be positive holomorphic semigroups acting on the Banach lattice $(\mathscr{R}, \geqslant)$ such that $S \geqslant T$. The following conditions conditions are equivalent:

1. $S>T$.

2. $\left\{S_{t}-T_{t}\right\}_{t>0}$ is BI-irreducible.

Similarly it follows as in Kishimoto and Robinson (1981) that if $S \geqslant T$ then every $S$-invariant Banach ideal is $T$-invariant and if $S>0$ then $S>T$ or $S=T$.

We conclude this discussion of positive semigroups on a Banach lattice with a remark concerning the modulus semigroup. Kubokawa (1975) has demonstrated that if $S$ is a $C_{0}$-semigroup acting on $L^{1}(x ; d \mu)$ then there exists a unique positive $C_{0}$-semigroup $\hat{S}$ such that

$$
\hat{S}_{t}|x| \geqslant\left|S_{t} x\right|, \quad t>0,
$$

for all $x \in L^{1}(x ; d \mu)$ and $\hat{S}$ is the smallest such semigroup (with respect to the order $\succcurlyeq$ ). The semigroup $\hat{S}$ is called the modulus semigroup of $S$; it is constructed from $S$ in two steps. First one defines the modulus $\left|S_{t}\right|$ of each $S_{t}$ by

$$
\left|S_{t}\right||x|=\sup _{|y| \leqslant|x|}\left|S_{t} y\right|
$$


and then extending to non-positive elements by linearity. Second one demonstrates that the limit

$$
\hat{S_{t}}=\lim _{n \rightarrow \infty}\left|S_{t / 2^{n}}\right|^{2^{n}}
$$

exists, defines a $C_{0}$-semigroup, and $\hat{S}_{t} \geqslant\left|S_{t}\right|$. (In fact Kubokawa uses a slightly different limit to define $\hat{S}$ but it follows by a slight extension of his arguments that $\hat{S}$ is also given by the above limit.)

In the general Banach lattice setting it is impossible to define a similar modulus semigroup; the construction relies either upon special $L^{1}$-, or $L^{\infty}$-, properties for the existence of $\left|S_{t}\right|$ or upon special features of the semigroup $S$. Nevertheless if $\hat{S}$ exists the following result relates irreducibility properties of $S$ and $\hat{S}$.

THEOREM 6. Let $S=\left\{S_{t}\right\}_{t>0}$ be a $C_{0}$-semigroup on an order complete Banach lattice $(\mathscr{G}, \geqslant)$ and assume the modulus semigroup $\hat{S}$ of $S$ exists. It follows that an order complete Banach ideal $(\mathfrak{Q}, \geqslant)$ is $S$-invariant if, and only if, it is $\hat{S}$-invariant.

Proof. Assume $(\mathbb{Q}, \geqslant)$ is $\hat{S}$-invariant and $x \in \mathbb{Q}$. But

$$
\hat{S}_{t}|x| \geqslant\left|S_{t} x\right|
$$

and $\hat{S}_{t}|x| \in \mathbb{Q}$. Therefore $S_{t} x \in \mathbb{Q}$ and $\mathbb{Q}$ is $S$-invariant.

Conversely if $(\mathbb{Q}, \geqslant)$ is $S$-invariant and $x \in \mathbb{Q}$ then

$$
\left|S_{t}\right||x|=\sup _{|v| \leqslant|x|}\left|S_{t} y\right| \text {. }
$$

But since $|y| \leqslant|x|$ one has $y \in \mathbb{Q}$ and hence $S_{t} y \in \mathbb{Q}$. Consequently $\left|S_{t}\right||x| \in \mathbb{Q}$ and $\hat{S}_{t}|x| \in \mathbb{Q}$ by order completeness. But

$$
\hat{S}_{t}|x| \geqslant\left|\hat{S}_{t} x\right|
$$

and hence $\tilde{S}_{t} x \in \mathbb{Q}$, that is, $\mathscr{Q}$ is $\hat{S}$-invariant.

REMARK. Order completeness is not used in the proof that $\hat{S}$ invariance implies $S$-invariance.

\section{Operator algebras}

In this section we discuss the extension of some of the foregoing results to positive semigroups acting on a $C^{*}$-algebra. We first demonstrate that Proposition 1 is valid in this alternative setting, albeit for different reasons, and subsequently we examine semigroups with the strict positivity property. 
Let $\mathfrak{A}$ denote a $C^{*}$-algebra, $\mathfrak{U}_{+}$the positive elements of $\mathfrak{U}$, and $\mathfrak{A}_{\text {sa }}$ the self-adjoint elements. The set $\mathfrak{A}_{s a}$ forms a real Banach space with respect to the norm of $\mathfrak{A}$ and this space is ordered by the relation $\geqslant$ induced by $\mathfrak{A}_{+} \cdot$ But $\left(\mathfrak{U}_{s a}, \geqslant\right)$ is a Banach lattice if, and only if the algebra $\mathfrak{A}$ is abelian (see, for example, Bratteli and Robinson (1979) Example 4.2.6). Thus for nonabelian $\mathfrak{A}$ the analysis of positive semigroups on $\left(\mathfrak{A}_{s a}, \geqslant\right)$ is quite different from the Banach lattice situation. Nevertheless one can introduce solid subsets, ideals, and Banach ideals, of $\mathfrak{A}_{s a}$ by the same definitions used in the Banach lattice context. The definition of $B I$-irreducibility also remains unchanged, as do the criteria of Proposition 1.

THEOREM 7. Let $S=\left\{S_{t}\right\}_{t>0}$ be a positive semigroup acting on the real ordered Banach space $\left(\mathfrak{U}_{s a}, \geqslant\right)$ associated with the $C^{*}$-algebra $\mathfrak{A}$. The following conditions are equivalent:

1. $S$ is BI-irreducible.

2. For each $x>0$ the ideal generated by $\left\{S_{t} x\right\}_{t>0}$ is dense in $\mathfrak{U}_{s a}$.

3. For each state $\omega$ on $\mathfrak{A}$ and each $x>0$ there exists $a t>0$ such that $\omega\left(S_{t} x\right)>0$.

REMARK. The theorem is valid for any set of positive operators $S$ with the property $S S \subseteq S$.

Proof. $1 \Rightarrow 2$. Assume Condition 2 is false. Thus there is an $x>0$ such that the Banach ideal $\mathcal{Q}$ generated by $\left\{S_{t} x\right\}_{t}>0$ is a strict subspace of $\mathfrak{A}_{s a}$. Therefore Condition 1 is false.

$2 \Rightarrow 1$. Assume that Condition 1 is false. Thus there exists a non-trivial $S$-invariant Banach ideal $\mathscr{Q} \subset \mathfrak{A}_{s a}$. Let $x$ be a non-zero positive element of $\mathcal{Q}$. It follows that the ideal generated by $\left\{S_{t} x\right\}_{t>0}$ cannot be dense in $\mathfrak{A}_{s a}$, that is, Condition 2 is false.

$2 \Rightarrow 3$. Assume Condition 3 is false. Thus $\omega\left(S_{t} x\right)=0$ for all $t>0$ and consequently $\omega(|y|)=0$ for all $y \in \mathfrak{U}_{s a}$ such that $|y| \leqslant S_{t} x$ for some $t>0$. But $|\omega(y)| \leqslant \omega(|y|)$ and hence $\omega(y)=0$. Therefore $\left.\omega\right|_{\mathbb{Q}}=0$ where $\mathcal{Q}$ is the Banach ideal generated by $\left\{S_{t} x\right\}_{t>0}$. Since $\omega \neq 0$ and $x \neq 0$ the ideal $\Theta$ is non-trivial and Condition 2 is false.

$3 \Rightarrow 2$. Assume Condition 2 is false. Thus there exists a non-zero $x \in \mathfrak{A}_{+}$such that the Banach ideal $\mathbb{Q}$ generated by $\left\{S_{t} x\right\}_{t>0}$ is non-trivial. Next consider the faithful representation

$$
\mathscr{H}=\bigoplus_{\omega \in E} \mathcal{K}_{\omega}, \quad \pi=\bigoplus_{\omega \in E} \pi_{\omega}
$$


where the direct sums extend over the state space $E_{\mathfrak{Y}}$ of $\mathscr{U}$ and $\left(\mathscr{K}_{\omega}, \pi_{\omega}\right)$ denotes the representation associated with $\omega \in E_{\mathfrak{A}}$. First it follows from Topping (1965) that the weak closure $\pi(\mathbb{Q})^{-}$of $\pi(\mathscr{Q})$ is a non-trivial Banach ideal of the self-adjoint part $M_{s a}$ of the weak closure $M$ of $\pi(\mathfrak{A})$. Second it follows from Proposition 3 of the foregoing reference that there exists a projection $E \in M_{s a}$ such that $\pi(\mathscr{Q})^{-}=E M_{s a} E$. But since $\mathscr{Q} \neq \mathfrak{A}_{s a}$ one has $E \neq I$. Thus if $\psi$ is a normalized vector in $(I-E) \mathscr{H}$ the associated state $\omega(A)=(\psi, A \psi)$ vanishes on $\mathbb{Q}$ and Condition 3 is false.

Next we consider the notion of a strictly positive semigroup acting on a $C^{*}$-algebra. For a general $C^{*}$-algebra this notion does not appear to be particularly appropriate. For example, if one demands that $\omega\left(S_{t} x\right)>0$ for all $\omega \in E_{\mathfrak{Y}}$, all non-zero $x \in \mathfrak{A}_{+}$, and all $t>0$, then one must have $\omega(x)>0$ for all $S$-invariant $\omega$ and all non-zero $x \in \mathfrak{A}_{+}$. But there are many examples which do not satisfy these latter conditions. Nevertheless strict positivity appears natural for a $W^{*}$-algebra. But since a strongly continuous positive semigroup on an abelian $W^{*}$-algebra is automatically uniformly continuous (Kishimoto and Robinson 1981) we now restrict our attention to $\sigma$-weakly continuous semigroups.

If $S=\left\{S_{t}\right\}_{t \geqslant 0}$ is a $\sigma$-weakly continuous semigroup over the $W^{*}$-algebra $M$, with $S_{0}=I$, we define $S$ to be positive whenever $S_{t} M_{+} \in M_{+}$for all $t>0$, and strictly positive if $\omega\left(S_{t} x\right)>0$ for all normal states $\omega$, all non-zero $x \in M_{+}$, and all $t>0$.

Strictly positive semigroups occur in the framework of equilibrium statistical mechanics at finite temperature. One way of constructing such semigroups is as follows.

Let $g \in G \mapsto \sigma_{g} \in$ Aut $M$ be a $\sigma$-weakly continuous action of the locally compact abelian group $G$ as *-automorphisms of the $W^{*}$-algebra $M$. Further let $t \in \mathbf{R}_{+} \mapsto \mu_{t}$ be a convolution semigroup (see Berg and Forst (1975)) over $G$ which is absolutely continuous with respect to the Haar measure $d g$ on $G$ and for which $\mu_{t}(G) \leqslant 1$. Finally assume the existence of a $G$-ergodic state $\omega(X)=(\Omega, X \Omega)$ over $M$ which is faithful, that is, $\Omega$ is both cyclic and separating for $M$. The positive contraction semigroup $S$ on $M$ is then defined by

$$
S_{t} x=\int d \mu_{t}(g) \sigma_{g}(x) .
$$

To prove that $S$ is strictly positive we argue by contradiction. Assume there exists a normal state $\omega^{\prime}$, a non-zero $x \in M_{+}$, and a $t>0$, such that $\omega^{\prime}\left(S_{t} x\right)=0$. Since $\mu_{t}(g)>0$ for $d g$-almost all $g$ we conclude that

$$
\int_{\Lambda} d g \omega^{\prime}\left(\sigma_{g}(x)\right)=0
$$


for any bounded subset $\Lambda \subseteq G$. By taking a limit over an appropriate net one obtains a mean value of $\sigma(x)$ and $G$-ergodicity and faithfulness of $\omega$ then imply that

$$
0=\lim _{\alpha} \frac{1}{\left|\Lambda_{\alpha}\right|} \int_{\Lambda_{\alpha}} d g \omega^{\prime}\left(\sigma_{g}(x)\right)=\omega(x)>0
$$

(see, for example, Bratteli and Robinson (1979) Theorems 4.3.22 and 4.3.23). This is a contradiction and hence $S$ is strictly positive.

The foregoing scheme occurs for the finite temperature pure phase equilibrium states of the ideal gases or for interacting quantum spin systems (see, for example, Bratteli and Robinson (1981b)). In these models one could take $G=\mathbf{R}^{\nu}$, or $\mathbf{Z}^{\nu}$, the group of space translations, and $d \mu_{t}(x)=(4 \pi t)^{-v / 2} \exp \left\{-x^{2} / 4 t\right\} d^{v} x$. It is perhaps worth noting that the corresponding semigroup is not strictly positive in the ground state nor in the corresponding $C^{*}$-dynamical system. These conclusions follow because the ground states $\omega$ are $S$-invariant but there are positive non-zero $x$ for which $\omega(x)=0$. Thus strict positivity discriminates between zero temperature and non-zero temperature behaviour.

One can describe the notion of strict positivity in terms of support projections. If $x \in M_{s a}$ the support projection $P(x)$ is defined as the smallest orthogonal projection in $M$ such that

$$
P(x) x=x=x P(x) .
$$

The following criterion follows immediately.

Proposition 8. Let $A$ be a positive bounded operator on the $W^{*}$-algebra $M$ and let $x$ be a non-zero positive element of $M$. The following conditions are equivalent:

1. $\phi(A x)>0$ for all normal states on $M$.

2. $P(A x)=1$.

Therefore $S=\left\{S_{t}\right\}_{t>0}$ is strictly positive if and only if $P\left(S_{t} x\right)=1$ for all non-zero positive $x$ and all $t>0$. Note also if $P\left(S_{t_{0}} x\right)=1$ then $P\left(S_{t} x\right)=1$ for all $t \geqslant t_{0}$. Thus strict positivity is equivalent to an instantaneous increase of $P\left(S_{t} x\right)$ to its maximum value as $t$ changes from zero to a non-zero value.

\section{Invariant states}

Throughout this final section we examine a pair $(\mathfrak{A}, S)$ where $\mathfrak{A}$ is a $C^{*}$-algebra and $S$ a semigroup acting on $\mathfrak{A}$ which is strongly positive in the sense that

$$
S_{t}\left(x^{*} x\right) \geqslant S_{t}(x) * S_{t}(x)
$$


for all $x \in \mathfrak{A}$ and all $t>0$. For simplicity we assume strong continuity of the map $t>0 \mapsto S_{t}$ but this is not essential. Moreover we note that $S$ is self-adjoint in the sense that

$$
S_{t}\left(x^{*}\right)=S_{t}(x)^{*} .
$$

The semigroups constructed in the previous section from *-automorphism groups and convolution measures satisfy the above properties; the strong positivity was established in Bratteli and Robinson (1981a).

Next we are interested in $S$-invariant states, that is, states $\omega$ over $\mathfrak{A}$ satisfying

$$
\omega\left(S_{t}(x)\right)=\omega(x)
$$

for all $t>0$ and $x \in \mathfrak{A}$. The $S$-invariant states form a convex set which is a weak * compact if $\mathfrak{A}$ possess an identity. An $S$-invariant state $\omega$ is defined to be $S$-ergodic if the only $S$-invariant positive functionals which it majorizes are of the form $\lambda \omega$ with $0 \leqslant \lambda<1$.

Strong positivity is of importance because it implies that

$$
\omega\left(S_{t}(x)^{*} S_{t}(x)\right) \leqslant \omega\left(S_{t}\left(x^{*} x\right)\right)=\omega\left(x^{*} x\right)
$$

for each $S$-invariant state. Hence if $\left(\mathcal{H}_{\omega}, \pi_{\omega}, \Omega_{\omega}\right)$ denotes the cyclic representation associated with $\omega$ then

$$
\left\|\pi_{\omega}\left(S_{t}(x)\right) \Omega_{\omega}\right\| \leqslant\left\|\pi_{\omega}(x) \Omega_{\omega}\right\| .
$$

Therefore one can define linear contraction operators $\hat{S}=\{\hat{S}\}_{t>0}$ on $\mathcal{H}_{\omega}$ by setting

$$
\hat{S}_{t} \pi_{\omega}(x) \Omega_{\omega}=\pi_{\omega}\left(S_{t}(x)\right) \Omega_{\omega}
$$

and then extending by continuity. Since $S$ is a semigroup it readily follows that the $\hat{S}_{t}$ form a semigroup, that is, $\hat{S}_{s} \hat{S}_{t}=\hat{S}_{s+t}$. Moreover $t \rightarrow \hat{S}_{t}$ is strongly continuous and $\hat{S}_{t} \Omega_{\omega}=\Omega_{\omega}$. We let $E_{\omega}$ denote the orthogonal projection on the subspace of $\mathcal{H}_{\omega}$ formed by the vectors invariant under all $\hat{S}_{t}$. Thus $\Omega_{\omega} \in E_{\omega} \mathcal{H}_{\omega}$.

It follows from the mean ergodic theorem (see, for example, Bratteli and Robinson (1979), Proposition 4.3.4 and Example 4.3.5) that

$$
\lim _{T \rightarrow \infty} \frac{1}{T} \int_{0}^{T} d t\left(\psi, \hat{S}_{t} \phi\right)=\left(\psi, E_{\omega} \phi\right)=\lim _{T \rightarrow \infty} \frac{1}{T} \int_{0}^{T} d t\left(\psi, \hat{S}_{t}^{*} \phi\right)
$$

for all $\phi, \psi \in \mathcal{H}_{\omega}$.

THEOREM 9. Let $S$ be a strongly positive, self-adjoint, $C_{0}$-semigroup of contractions acting on the $C^{*}$-algebra $\mathfrak{A}$. Further let $\omega$ be an $S$-invariant state over $\mathfrak{A}$ with associated cyclic representation $\left(\mathcal{K}_{\omega}, \pi_{\omega}, \Omega_{\omega}\right)$. Define the $C_{0}$-semigroup of contractions $\hat{S}$ on $\mathcal{K}_{\omega}$ such that

$$
\hat{S}_{t} \pi_{\omega}(x) \Omega_{\omega}=\pi_{\omega}\left(S_{t}(x)\right) \Omega_{\omega}, \quad x \in \mathfrak{H}
$$


and let $E_{\omega}$ denote the orthogonal projection on the $\hat{S}$-invariant subspace of $\mathcal{H}_{\omega}$. The following conditions are equivalent:

1. $\lim _{T \rightarrow \infty}(1 / T) \int_{0}^{T} d t \omega\left(x S_{t}(y)\right)=\omega(x) \omega(y)$,

2. $E_{\omega}$ has rank one,

and imply

3. $\omega$ is S-ergodic.

Moreover if

4. $\lim _{T \rightarrow \infty}(1 / T) \int_{0}^{T} d t\left(\phi, \pi_{\omega}\left(\left[S_{t}(x), y\right]\right) \Omega_{\omega}\right)=0$

for all $x, y \in \mathfrak{A}$ and $\phi \in \mathcal{H}_{\omega}$ then the first three conditions are equivalent. $\hat{S}$.

Proof. $1 \Leftrightarrow 2$. This is an algebraic restatement of the mean ergodic theorem for

$2 \Rightarrow 3$. Let $\omega^{\prime}$ be an $S$-invariant state which is dominated by $\omega$. It follows that there exists a positive $A \in \pi_{\omega}(\mathfrak{H})^{\prime}$ with $\|A\| \leqslant 1$ and $\omega(A)=1$ such that

$$
\omega^{\prime}(x)=\left(\Omega_{\omega}, A \pi_{\omega}(x) \Omega_{\omega}\right) .
$$

But since $\omega^{\prime}$ is $S$-invariant this gives

$$
\left(\Omega_{\omega}, A \pi_{\omega}(x) \Omega_{\omega}\right)=\left(\Omega_{\omega}, A \hat{S}_{t} \pi_{\omega}(x) \Omega_{\omega}\right) .
$$

Therefore by taking mean values one obtains

$$
\begin{aligned}
\left(\Omega_{\omega}, A \pi_{\omega}(x) \Omega_{\omega}\right) & =\left(\Omega_{\omega}, \pi_{\omega}(x) \Omega_{\omega}\right)\left(\Omega_{\omega}, A \Omega_{\omega}\right) \\
& =\left(\Omega_{\omega}, \pi_{\omega}(x) \Omega_{\omega}\right) .
\end{aligned}
$$

$4+3 \Rightarrow 2$. For each $x \in \mathfrak{A}$ define the linear functional $\phi_{x}$ by

$$
\begin{aligned}
\phi_{x}(y) & =\lim _{T \rightarrow \infty} \frac{1}{T} \int_{0}^{T} d t \omega\left(y S_{t}\left(x^{*} x\right)\right) \\
& =\left(\Omega_{\omega}, \pi_{\omega}(y) E_{\omega} \pi_{\omega}\left(x^{*} x\right) \Omega_{\omega}\right) .
\end{aligned}
$$

(The second identification follows from the mean ergodic theorem.) But using Condition 4 one has

$$
\phi_{x}\left(y^{*} y\right)=\lim _{T \rightarrow \infty} \frac{1}{T} \int_{0}^{T} d t \omega\left(y^{*} S_{t}\left(x^{*} x\right) y\right) \geqslant 0 .
$$

Thus $\phi_{x}$ is positive. Moreover the identification (4) demonstrates that

$$
\begin{aligned}
\phi_{x}\left(S_{t}(y)\right) & =\left(\pi_{\omega}\left(S_{t}(y)^{*}\right) \Omega_{\omega}, E_{\omega} \pi_{\omega}\left(x^{*} x\right) \Omega_{\omega}\right) \\
& =\left(\Omega_{\omega}, \pi_{\omega}\left(y^{*}\right) \hat{S}_{t}^{*} E_{\omega} \pi_{\omega}\left(x^{*} x\right) \Omega_{\omega}\right) \\
& =\phi_{x}(y),
\end{aligned}
$$

that is, $\phi_{x}$ is $S$-invariant. But from (2) one sees that

$$
\phi_{x}\left(y^{*} y\right) \leqslant \omega\left(y^{*} y\right)\|x\|^{2},
$$


that is, $\phi_{x}$ is dominated by $\omega$. Therefore Condition 3 implies that

$$
\phi_{x}(y)=\lambda \omega(y)
$$

where $0 \leqslant \lambda \leqslant\|x\|^{2}$. Setting $y$ equal to the identity (or strongly approximating $\Omega_{\omega}$ by a sequence $\left\{\pi_{\omega}\left(y_{n}^{*}\right) \Omega_{\omega}\right\}_{n \geq 1}$ if $\mathscr{A}$ does not possess an identity) one finds

$$
\lambda=\left(\Omega_{\omega}, \pi_{\omega}\left(x^{*} x\right) \Omega_{\omega}\right)=\omega\left(x^{*} x\right) .
$$

Finally combining (1) and (3) with this identification one obtains the identity

$$
\begin{aligned}
\left(\Omega_{\omega}, \pi_{\omega}(y) E_{\omega} \pi_{\omega}\left(x^{*} x\right) \Omega_{0}\right) & =\omega(y) \omega\left(x^{*} x\right) \\
& =\left(\Omega_{\omega}, \pi_{\omega}(y) \Omega_{\omega}\right)\left(\Omega_{\omega}, \pi_{\omega}\left(x^{*} x\right) \Omega_{\omega}\right) .
\end{aligned}
$$

Thus $E_{\omega}$ is the orthogonal projection onto $\Omega_{\omega}$.

A result similar to Theorem 9 , for faithful $\omega$, has previously been obtained by Frigerio (1977) and this result has subsequently been extended by Robinson (1982) and Batty (1982).

\section{Acknowledgements}

The first author was supported by an ARGC Fellowship during the year 1980 at the University of New South Wales when the major part of this work was accomplished. The second author was partially supported by an SRC Research Grant at Bedford College, London University, where the work was completed.

\section{References}

C. J. K. Batty (1982), 'Invariant states for strongly positive operators on $C^{*}$-algebras', Univ. of Edinburgh $\bar{p}$ reprint.

C. Berg and G. Forst (1975), Potential theory on locally compact abelian groups (Springer-Verlag, Berlin, Heidelberg, New York).

O. Bratteli, A. Kishimoto, and D. W. Robinson (1980), 'Positivity and monotonicity properties of $C_{0}$-semigroups. I’, Comm. Math. Phys. 75, 67-84; II, Comm. Math. Phys. 75, 85-101.

O. Bratteli and D. W. Robinson (1979), Operator algebras and quantum statistical mechanics, Vol 1 (Springer-Verlag, Berlin, Heidelberg, New York),

O. Bratteli and D. W. Robinson (1981a), 'Positive $C_{0}$-semigroups on $C^{*}$-algebras', Math. Scand. to appear.

O. Bratteli and D. W. Robinson (198lb), Operator algebras and quantum statistical mechanics, Vol. 2 (Springer-Verlag, Berlin, Heidelberg, New York).

A. Frigerio, (1977), 'Quantum dynamical semigroups and approach to equilibrium', Lett. Math. Phss. 2, 33-38.

A. Kishimoto and D. W. Robinson (1981), 'Subordinate semigroups and order properties', J. Austral. Math. Soc. Ser. A, to appear.

Y. Kubokawa (1975). 'Ergodic theorem for contraction semi groups', J. Math. Soc. Japan 27. $184-193$. 
D. W. Robinson (1982), 'Strongly positive semigroups and faithful invariant states', Comm. Math. Phys., to appear.

H. H. Schaefer (1974), Banach lattices and positive operators (Springer-Verlag, Berlin, Heidelberg, New York)

B. Simon (1973), 'Ergodic semigroups of positivity preserving self-adjoint operators', J. Functional Analysis 12, 335-339.

D. M. Topping (1965), 'Jordan algebras of self-adjoint operators', Mem. Amer. Math. Soc. 53.

\author{
Department of Pure Mathematics \\ University of New South Wales \\ Kensington, N.S.W. 2033 \\ Australia
}

